\title{
Ceftaroline Fosamil for the Treatment of Community- Acquired Pneumonia: from FOCUS to CAPTURE
}

\author{
Joseph J. Carreno • Thomas P. Lodise
}

To view enhanced content go to www.infectiousdiseases-open.com

Received: June 13, 2014 / Published online: September 6, 2014

(C) The Author(s) 2014. This article is published with open access at Springerlink.com

\section{ABSTRACT}

Ceftaroline fosamil (ceftaroline hereafter) is the latest addition to the armamentarium for the treatment of patients with community-acquired pneumonia (CAP). It is currently approved by the Food and Drug Administration (FDA) for community-acquired bacterial pneumonia (CABP), which is a recent FDA indication that centers on individuals with documented bacterial pneumonias that arise in the community setting. The purpose of this review is to summarize and discuss the major findings from the Phase III CAP clinical trials as well as the clinical experience with ceftaroline among patients with CAP in the "Ceftaroline Assessment Program and Teflaro ${ }^{\circledR}$ Utilization Registry" (CAPTURE). In its two Phase III CAP trials, ceftaroline was compared to ceftriaxone

Electronic supplementary material The online version of this article (doi:10.1007/s40121-014-0036-8) contains supplementary material, which is available to authorized users.

J. J. Carreno ( $₫)$. T. P. Lodise

Department of Pharmacy Practice, Albany College of Pharmacy and Health Sciences, School of Pharmacy and Pharmaceutical Sciences, 106 New Scotland Ave, Albany, NY 12208, USA

e-mail: joseph.carreno@acphs.edu among adults with radiographically confirmed CAP requiring hospitalization who were classified as Pneumonia Outcomes Research Team (PORT) risk class III or IV. Among patients with CAP, clinical success at test of cure was $84.3 \%$ vs $77.7 \%$ (difference $6.6 \%, 95 \%$ confidence interval [CI]: $1.6-11.8 \%)$ in those treated with ceftaroline and ceftriaxone, respectively, across the two Phase III clinical trials. Among patients with a culture-confirmed CABP, day 4 response rates were numerically higher, albeit non-significant, among patients that received ceftaroline vs. ceftriaxone $(69.5 \%$ for ceftaroline vs. $59.4 \%$ for ceftriaxone, difference $10.1 \%, 95 \% \mathrm{CI},-0.6 \%$ to $20.6 \%$ ). The efficacy of ceftaroline is supported by realworld observational data from CAPTURE for patients with both CAP and CABP. In addition, the CAPTURE program afforded an opportunity to assess the outcomes of patients who were excluded or limited in the original Phase III trials in a non-comparative fashion. These underrepresented patient populations with CAP included: patients that received prior antibiotics, patients in the ICU, patients with severe renal dysfunction, and those with methicillin-resistant Staphylococcus aureus 
(MRSA) isolated from respiratory or blood culture. As CAPTURE is a retrospective, noncomparator convenience sample registry, all the findings need to be interpreted with caution.

Keywords: Ceftaroline fosamil; Communityacquired pneumonia; Efficacy; Infection

\section{INTRODUCTION}

Ceftaroline fosamil (ceftaroline hereafter) is the latest addition to the armamentarium for the treatment of patients with community-acquired pneumonia (CAP), including those with a documented bacterial pneumonia. It is currently approved by the FDA for the treatment of adults with community-acquired bacterial pneumonia (CABP) caused by susceptible isolates of the following Grampositive and Gram-negative microorganisms: Streptococcus pneumoniae (including cases with concurrent bacteremia), Staphylococcus aureus (methicillin-susceptible isolates only), Haemophilus influenzae, Klebsiella pneumoniae, Klebsiella oxytoca, and Escherichia coli [1]. The purpose of this review is to summarize the major efficacy and effectiveness findings of ceftaroline from the Phase III CAP clinical trials [2-4] and from the "Ceftaroline Assessment Program and Teflaro ${ }^{\circledR}$ Utilization Registry" (CAPTURE) [5-10].

When reviewing the Phase III "efficacy" and post-marketing "effectiveness" data for ceftaroline, it is important to appreciate the distinction between CAP and CABP [11, 12]. Both CAP and CABP are acute infections of the lower respiratory tract (pulmonary parenchyma) among patients not hospitalized or residing in a long-term care facility for $\geq 14$ days before the onset of symptoms [11-14]. The difference between CAP and CABP lies in their etiology.
Community-acquired pneumonia can be caused by bacterial pathogens and certain respiratory viruses. Its etiology is often unknown at clinical presentation $[13,14]$. In contrast, CABP is the recent Food and Drug Administration (FDA) designation to identify individuals with a documented bacterial pneumonia [11, 12]. The FDA decided to make this distinction to more appropriately identify patients who are most likely to have pneumonia of bacterial etiology and who would benefit most from antimicrobial therapy $[15,16]$. This is a critical distinction, since the etiology of CAP is often unknown in both clinical trials and clinical practice $[2-4,13$, $14,17]$. In clinical trials, bacterial pathogens are identified in only $25 \%$ of cases [2, 4, 17]. In practice, a microbiological diagnosis in CAP occurs in less than $10 \%$ of cases [18]. Thus, although it is approved by the FDA for CABP, much of its use in the real-world setting is for CAP since the bacterial etiology is not frequently established [18]. As such, it is important to understand the efficacy and effectiveness of ceftaroline in these two distinct yet related disease states when evaluating its potential for use in clinical practice.

\section{METHODS}

Studies included were the CAP FOCUS trials (NCT00621504 and NCT00509106) and studies evaluating effectiveness of ceftaroline in the treatment of CAP and CABP from the CAPTURE registry.

\section{Compliance with Ethics}

The analysis in this article is based on previously conducted studies, and does not involve any new studies of human or animal subjects performed by any of the authors. 


\section{CEFTAROLINE}

\section{Major Findings from Phase III Clinical Trials for CAP}

Although ceftaroline is indicated by the FDA for CABP, its two randomized, double-blind, international multicenter Phase III trials were designed and initiated before the recent changes in the FDA guidance for CABP. As such, the Phase III trials were designed originally to compare ceftaroline $600 \mathrm{mg}$ intravenously ( $1 \mathrm{~h}$ infusions) twice daily versus ceftriaxone $1 \mathrm{~g}$ intravenously daily among hospitalized adults with CAP (CeFtarOline Community-acquired PneUmonia Trial VerSus Ceftriaxone in hospitalized patients [FOCUS] 1 and FOCUS 2; ClinicalTrials.gov identifiers NCT00621504 and NCT00509106) [2-4]. These were non-inferiority trials and the two studies used nearly identical designs and methods. Both enrolled adults with radiographically confirmed CAP requiring hospitalization and IV antimicrobial therapy and who were classified as Pneumonia Outcomes Research Team (PORT) risk class III or IV [19]. Patients who were admitted to an ICU or were candidates for outpatient therapy with an oral antimicrobial were excluded in both studies. Finally, both studies excluded patients who had confirmed or suspected methicillin-resistant $S$. aureus (MRSA) infection because of the inactivity of ceftriaxone against this pathogen. There was, however, one notable difference between studies. In FOCUS 1, patients received two oral doses of clarithromycin $500 \mathrm{mg}$ as adjunctive therapy on day 1 , consistent with the American Thoracic Society/Infectious Diseases Society of America (ATS/IDSA) CAP clinical management guidelines [3]. No empirical macrolide use was permitted in FOCUS 2.

Across FOCUS 1 and 2, over 1,200 hospitalized adults with CAP were enrolled. Consistent with most randomized clinical trials of this size, treatment groups were highly comparable at baseline. Patients were predominantly white (93\%) and male (63\%), with approximately $50 \%$ of the patients over the age of 65 . The distribution of PORT risk was $62.9 \%$ in class III and $37.1 \%$ in class IV in FOCUS 1 , and $60.7 \%$ class III and $39.3 \%$ class IV in FOCUS 2. Not surprisingly, S. pneumoniae and methicillin-susceptible $S$. aureus (MSSA) were the most commonly isolated pathogens in both studies: $36.4 \%$ and $15.7 \%$, respectively, in FOCUS 1 , and $44.1 \%$ and $18.6 \%$, respectively, in FOCUS 2 [2].

Overall, the results demonstrated that ceftaroline had comparable efficacy to ceftriaxone. In the clinically evaluable integrated population, test of cure (TOC) was evaluated 8-15 days after last dose of study drug. Clinical success at the TOC visit was $84.3 \%$ among patients that received ceftaroline versus $77.7 \%$ among patients who received ceftriaxone (difference 6.6\%, 95\% confidence interval (CI), 1.6-11.8\%). In the integrated modified intent to treat efficacy population (mITTE), $82.6 \%$ of ceftaroline-treated patients achieved clinical cure compared with $76.6 \%$ of ceftriaxonetreated patients (difference 6.0\%, 95\% CI, 1.4-10.7\%). Among patients with $S$. pneumoniae identified as a baseline pathogen $(n=139)$, the clinical cure rate was $85.7 \%$ in the ceftaroline group and $69.5 \%$ in the ceftriaxone group ( $p$ value not reported). For patients with MSSA identified at baseline $(n=55)$, the clinical cure rates were $72.0 \%$ for ceftaroline and $60.0 \%$ for ceftriaxone, respectively ( $p$-value not reported). 


\section{Major Findings from Phase III Clinical Trials for CABP}

As mentioned above, the FDA updated its guidance as ceftaroline was proceeding through the regulatory process $[12,20]$. Rather than focusing on CAP and TOC assessments for evaluating clinical response and determining non-inferiority, the FDA endorsed the use of an early clinical response endpoint between study days 3-5 based on clinical symptom improvement and stabilization of vital signs among patients with a documented CABP. Given the change in guidance, a post hoc analysis of day 4 response rates was performed among patients enrolled in the FOCUS studies who met the following inclusion criteria: received at least one dose of study drug, had CAP that met radiographic criteria, had at least one symptom at baseline, and had one or more acceptable baseline typical pathogens [21]. This change in endpoint is clinically relevant because clinicians are unlikely to wait until the end of therapy to assess clinical response in practice. Rather, clinicians' early assessment of clinical response is more likely to guide therapy and subsequent therapy changes. Hence, the updated trial design improved the external validity of the clinical findings. The early response endpoint is also consistent with the definition of a patient eligible for hospital discharge in the ATS/IDSA CAP guidelines [14].

In the combined analysis of FOCUS 1 and FOCUS 2, response rates at day 4 were $69.5 \%$ for ceftaroline and $59.4 \%$ for ceftriaxone (difference $10.1 \%, 95 \% \mathrm{CI},-0.6 \%$ to $20.6 \%$ ). Among patients infected with $S$. pneumoniae, day 4 response rates were statistically significantly higher with ceftaroline (73\%, $54 / 74)$ relative to ceftriaxone $(56 \%, 42 / 75)$ (difference 17\%, 95\% CI, 1.4-31.6\%; $p=0.03$ ). The response rates at day 4 for patients with
MSSA were 58.3\% (14/24) for those treated with ceftaroline and $54.8 \%(17 / 31)$ for ceftriaxone (difference $3.5 \%, 95 \% \mathrm{CI},-24.7 \%$ to $26.2 \%$ ) [21].

\section{Interpretation of Findings from Phase III Studies}

Collectively, these findings suggest that, with regard to efficacy, ceftaroline is a non-inferior alternative to ceftriaxone for the treatment of PORT III and IV hospitalized patient with CABP. The study findings also indicate that ceftaroline has utility in the empiric treatment of noncritically hospitalized patients with CAP. The comparative data were highly notable for patients with culture-confirmed $S$. pneumoniae, the most common cause of CABP. The more favorable early response at day 4 with ceftaroline among those with cultureconfirmed $S$. pneumoniae is suggestive of a more accelerated time to clinical stability, and hence, hospital discharge. Although the definitive reason in response rates at day 4 and TOC among patients with culture-confirmed $S$. pneumoniae are unclear, the differences in outcomes may be explained by ceftaroline's enhanced affinity for penicillin-binding protein (PBP) 1a, 2a, 2b, and 2x as compared to ceftriaxone [22]. In particular, increased affinity for PBP2x increases in vitro efficacy against penicillin-intermediate, penicillinresistant, and multidrug-resistant $S$. pneumoniae (MDRSP) [23]. However, the clinical relevance is unclear as there were only eight documented cases of MDRSP in the FOCUS trials. Of note, the study required that only patients with ceftriaxone-susceptible bacteria were included in the microbiological evaluable population. As such, no inferences regarding the efficacy of ceftaroline relative to ceftriaxone for ceftriaxone intermediate- and 
resistant-Streptococcus pneumoniae isolates can be gleaned from the Phase III trials.

Despite the positive findings, the FOCUS trials were not without limitations. Specifically, critically ill patients in the ICU, those with culture-confirmed MRSA pneumonia, and those with severe renal dysfunction were excluded. These patients are important special populations because they may more accurately describe the patient population who may benefit from treatment with ceftaroline. Consequently, it is vital to examine the realworld effectiveness of any new antibiotic as it is used in a broader range of patients among patients with both CAP and CABP.

\section{Experience with Ceftaroline in the CAPTURE Registry}

CAPTURE is a multicenter, retrospective registry of patients receiving ceftaroline dosed per package insert recommendations (i.e., $600 \mathrm{mg}$ intravenously twice a day or dose adjusted for renal dysfunction) for the treatment of CABP and CAP. The data generated from CAPTURE provide critical insights into the real-world effectiveness of ceftaroline for both CABP and CAP [5-10]. It provides clinical outcome data on patient populations and bacterial pathogens not well represented or excluded in the Phase III clinical trials (i.e., MRSA). The CAPTURE program also provides the opportunity to collect data on outcomes not traditionally examined in Phase III trials, like hospital length of stay and healthcare costs.

\section{CAPTURE: Year One and Two}

The first 2 years of CAPTURE examined clinical effectiveness and safety among patients treated with ceftaroline for CAP. In the first year of the CAPTURE registry (August 2011 to August 2012), data were available on 272 patients with CAP from 30 study centers [10, 24]. At the time of the year one analysis, the cohort well reflected a patient population commensurate with inpatients being treated for CAP. Most patients were older (mean [SD] age: 63.6 [17.9]), males (54\%) with at least one comorbidity (76\%). The most prevalent comorbidities included structural lung disease (40\%), smoking (28\%), recent pneumonia (24\%), and congestive heart failure (19\%). Overall clinical success, defined as no need for further antibiotics or clinical improvement with switch to oral antibiotics, was $77 \%$. Patients' mean (SD) length of therapy (LOT) was 6.3 (4.7) days. Most patients were discharged to home $(58 \%)$ or another healthcare facility (38\%). Patients seldom discontinued treatment due to adverse events $(n=6,2 \%)$. These findings suggest that in a real-world setting, ceftaroline has similar effectiveness as compared to that observed in the Phase III clinical trials.

Several caveats should be noted when interpreting these findings. First, $84 \%$ of patients received antibiotics prior to ceftaroline. The most commonly used antibiotics were other cephalosporins (35\%), glycopeptides (34\%), quinolones (32\%), macrolides (25\%), and penicillins (21\%). However, the authors note that clinical success was similar in patients receiving prior antibiotics as compared to those without prior antibiotics (77\% and $75 \%$, respectively). In addition, it is important to recognize that less than one-half of patients received ceftaroline as monotherapy (37\%). Patients that received combinations of ceftaroline often received quinolones (21\%), macrolides (20\%), and glycopeptides (13\%). Concurrent utilization of additional antibiotics may lead to overestimation of the treatment effect of ceftaroline. Lastly, the failure to note differences within subgroups may be due to limited power. 
As the CAPTURE registry was expanded, the outcomes of patients with CAP were reexamined [5]. Between August 2011 and February 2013, 528 patients with CAP were enrolled and eligible for evaluation. The mean age was 63.8 years, over half the population was female, and $60.8 \%$ were white. The majority (76.5\%) had relevant medical history including structural lung disease (43.2\%), prior pneumonia (25.4\%), GERD (24.1\%), and CHF (21.4\%). Similar to the first CAPTURE analysis of patients with CAP, $31.4 \%$ patients were past or present smokers. The majority of patients used ceftaroline as non-first line therapy ( $n=445, \quad 84.3 \%)$. Monotherapy was still infrequent $(n=28,33.7 \%)$ among patients that received ceftraroline as first-line therapy. Among those who received ceftaroline first line, the mean (median) LOT was 5.8 (5.0) days and the mean (median) LOS was 11.8 (7.0) days. In contrast, mean (median) LOT was 6.2 (5.0) and the mean (median) LOS was 13.4 (9.0) days ( $p$-value not reported) in those receiving ceftaroline not as first-line therapy. The mean (median) total hospital charges were $\$ 93,183$ $(\$ 44,741)$ and $\$ 106,076(\$ 53,825)$ for first-line and non-first line cohorts, respectively. Irrespective of receiving first- or non-first line therapy with ceftaroline, the majority of patients were discharged to home $(64.8 \%)$ or to another care facility (16.2\%).

These data suggest that there may be a cost benefit from utilizing ceftaroline as first-line therapy. Overall, those who received ceftaroline as first-line therapy tended to have shorter lengths of stays and lower total hospital charges. However, there are several important considerations with these data. The findings were descriptive in nature and multivariate statistics were not performed. Therefore, it is unclear if unequal distribution of baseline characteristics or unmeasured confounders may have affected the study results. In the patients receiving ceftaroline as non-first line therapy it is possible that these patients were switched from inactive or insufficient therapy. These delays in time to appropriate therapy may account for some of the observed differences between study groups. Hence, prior to adopting ceftaroline as a first-line therapy for the purpose of cost savings, additional research is needed.

\section{Special Populations Within CAPTURE}

In addition to validating findings from the FOCUS trials, CAPTURE also examined outcomes in previously unexamined special populations. In FOCUS, critically ill patients in intensive care units were excluded [24]. However, critically ill patients were eligible for enrollment in CAPTURE. In the first CAPTURE evaluation of patients with CAP, 99 (36\%) patients were admitted to the ICU and their cure rate was $67 \%$. These data suggest that there may be a role for ceftaroline in treatment of CAP among patients admitted to the ICU.

The CAPTURE registry also provided a unique opportunity to examine ceftaroline use with and without vancomycin for patients with CAP [24]. For this analysis, data were available on 175 patients with CAP. Among these patients, $77 \% \quad(n=134)$ received ceftaroline monotherapy and $23 \% \quad(n=41)$ received ceftaroline plus vancomycin. Baseline demographics were similar to previous CAPTURE evaluations. Patients receiving ceftaroline monotherapy and combination therapy had a similar average (median) LOT (6.4 [6] vs 6.8 (6) days, respectively, $p$-value not reported). The mean total hospital length of stay was longer in the combination group (20.9 vs. 14.6 days, $p$-value not reported). Numerically similar proportions of patients receiving monotherapy and combination therapy were discharged to home $(55 \%$ vs. 
$41 \%, p$-value not reported) or another care facility ( $40 \%$ vs. $44 \%$, $p$-value not reported). Four patients expired in the study period, all of which were in the combination group. Although these data may suggest that the addition of vancomycin to ceftaroline for CAP does not improve outcomes, it is important to note that more patients in the combination therapy group were admitted to the ICU. Conversely, ceftaroline monotherapy was more common in the general practice units (66\%). This potential selection bias may have skewed the results in favor of ceftaroline monotherapy but more data are needed in each patient care setting (ICU vs. non-ICU) before definitive conclusions can be made.

Within the FOCUS trials, patients with severe renal dysfunction (CrCL $<30 \mathrm{~mL} / \mathrm{min}$ ) were excluded $[3,4]$. The CAPTURE registry has provided an opportunity to study a small cohort (26 patients) with renal insufficiency (baseline serum creatinine $>1.8 \mathrm{mg} / \mathrm{dL}$ ) [7]. The majority of patients were male $(n=15,58 \%)$, the mean (SD) age was 67.9 years, and average BMI was $28.2 \mathrm{~kg} / \mathrm{m}^{2} \quad$ [2]. The most prevalent comorbidities among patients with renal impairment and CAP were GERD $(n=8,31 \%)$, history of smoking $(n=7,27 \%)$, and CHF $(n=6,23 \%)$. Most patients $(n=19,73 \%)$ were treated in general practice units. Prior antibiotics were again common; the most frequent antibiotics received prior to ceftaroline were glycopeptides (31\%), macrolides (31\%), and quinolones (27\%). Concurrent antibiotics were also commonplace (65\%). The outcomes among patients with renal insufficiency were generally consistent with the overall cohort. The overall clinical cure rate was $81 \%$ and the mean (standard deviation) LOT was 5.8 (3.1) days. Most patients were sent home (62\%) after hospital discharge. These findings add substantially to the literature regarding the effectiveness of ceftaroline in patients with renal dysfunction. However, consistent with the other subgroup analyses, the limited sample size and the potential for selection bias necessitate the need for additional verification prior to routine use in clinical practice.

Another area of interest for clinicians is the ability of ceftaroline to treat MRSA CABP. Patients with MRSA CABP were specifically excluded from the FOCUS trials due to the inactivity of ceftriaxone against MRSA [2-4]. CAPTURE has afforded an opportunity to examine the use of ceftaroline for patients with CABP with positive cultures for MRSA [6]. At the time of abstract presentation in 2013, there were a total of 39 patients with CABP with positive cultures for MRSA in CAPTURE. With regard to culture sites, MRSA was isolated from both blood and respiratory samples in three patients (8\%), respiratory samples only in 28 patients $(72 \%)$, and blood samples only in 8 patients $(21 \%)$. The cohort of patients with CABP with a positive MRSA culture was predominately male $(n=25,64 \%)$ and the mean (SD) age was 59.0 (16.6) years. Similar to the other subgroups examined, comorbidities were highly prevalent. Thirty-three patients (85\%) had comorbidities including structural lung disease (56.4\%), GERD (33.3\%), history of smoking (25.6\%), prior pneumonia (20.5\%), and CHF (18.0\%). There was an equal proportion of patients admitted to intensive care units and general practice units (51\% vs. $49 \%)$. Nearly all patients $(n=36,92 \%)$ received prior antibiotics before initiation of ceftaroline. Glycopeptides, cephalosporins, and penicillins were the most commonly used prior antibiotics $(67 \%, 31 \%$, and $31 \%$, respectively). Half the patients $(n=20)$ received ceftaroline as monotherapy, while the remainder received concurrent gylcopeptides (28\%), quinolones 
(15\%), and macrolides (8\%). Patients were treated for a mean (range) of 7.3 days (range 1-30 days). The incidence of clinical success was $62 \%(n=24)$. Similar to other investigations, clinical success was greater in those admitted to the general practice units relative to the ICU ( $74 \%$ vs. $50 \%$, respectively). Source of pathogen isolation did not affect clinical cure (respiratory: 61\%, blood: 64\%). Ceftaroline monotherapy was associated with higher rates of clinical success as compared to combination therapy (75\% vs 47\%). Among those with a clinical failure, two patients were transferred to hospice care and one patient had a lobectomy due to a lung abscess. A high proportion of patients were discharged home (46\%), while fewer were discharged to another care facility (44\%).

\section{Considerations with CAPTURE and Future Directions}

Overall, the clinical response rates observed among patients in the CAPTURE registry were consistent with those observed among patients in the FOCUS trials. These findings also highlighted the potential utility of ceftaroline for the treatment of patients with CAP among populations that were excluded from the phase III clinical trials. However, several caveats should be noted when interpreting these findings. First, CAPTURE is a non-comparator, convenience sample, observational registry. As such, all findings need to be interpreted with caution prior to full adoption into clinical practice. This is especially true for patients with CABP due to MRSA. The ability to effectively use ceftaroline for patients with CABP due to MRSA will be better elucidated upon completion of the current ongoing perspective clinical trial that is assessing its efficacy in patients with CABP due to MRSA. Second, it is difficult to fully discern the effectiveness of ceftaroline in CAPTURE as the combination therapy was common and sample size was limited (increasing the potential for type II error) across many specialized population assessed. Third, the role of prescribing bias and confounding on the observed outcomes cannot be elucidated clearly due to the sampling method and non-comparative nature of the registry.

As the data in CAPTURE registry expands, it would be highly beneficial to ascertain ceftaroline's "real-world" effectiveness as the number of patients that receive first-line ceftaroline monotherapy across important specialized patient populations increases. It would also be advantageous to include a comparator arm to the registry to measure the effectiveness of ceftaroline relative to other commonly used antibiotic regimens for CAP. As part of these comparator studies, it is important to compare readmission rates between patients that receive different therapies. This is especially relevant in light of the Patient Protection and Affordable Care Act [25] which will trigger withholding of reimbursement as a penalty for higher-thanexpected readmission rates among Medicaid patients with pneumonia. Finally, it would also be useful to expand the CAPTURE program to examine the effect of ceftaroline use on antibiotic resistance rates within a given institution. Third-generation cephalosporin use within health systems has been linked to increase prevalence of extended spectrum beta-lactamase (ESBL)-producing organisms. Given the similar spectrum of ceftaroline to ceftriaxone, it would be prudent to evaluate the association of ceftaroline use with prevalence of ESBL-producing organisms.

\section{CONCLUSIONS}

Community-acquired bacterial pneumonia continues to be a grave public health concern. 
Ceftaroline is a new addition to our antibiotic treatment arsenal for patients with both CAP and CABP. Data from clinical trials suggest that ceftaroline is non-inferior to ceftriaxone and has a reasonable safety profile [2-4]. These findings have been supported by real-world observational data from CAPTURE [5-10]. In addition, the CAPTURE program afforded an opportunity to assess the outcomes of patients who were excluded or limited in the original Phase III trials patients in a non-comparative fashion. The CAPTURE registry has provided valuable insights into ceftaroline use in special populations including the elderly, critically ill, those with renal dysfunction, and those with MRSA CABP. As CAPTURE is a retrospective, non-comparator convenience sample registry, all the findings need to be interpreted with caution.

\section{ACKNOWLEDGMENTS}

No funding or sponsorship was received for this study or publication of this article. All named authors meet the ICMJE criteria for authorship for this manuscript, take responsibility for the integrity of the work as a whole, and have given final approval for the version to be published.

Conflict of interest. TPL is a consultant, speaker, and grant recipient for Forest. JJC declares no conflict of interest.

Compliance with ethics. The analysis in this article is based on previously conducted studies, and does not involve any new studies of human or animal subjects performed by any of the authors.

Open Access. This article is distributed under the terms of the Creative Commons
Attribution Noncommercial License which permits any noncommercial use, distribution, and reproduction in any medium, provided the original author(s) and the source are credited.

\section{REFERENCES}

1. Teflaro (ceftaroline fosamil) [full prescribing information]. New York: Forest Laboratories; 2010.

2. File TM Jr, Low DE, Eckburg PB, et al. Integrated analysis of FOCUS 1 and FOCUS 2: randomized, doubled-blinded, multicenter phase 3 trials of the efficacy and safety of ceftaroline fosamil versus ceftriaxone in patients with community-acquired pneumonia. Clin Infect Dis. 2010;51:1395-405.

3. File TM Jr, Low DE, Eckburg PB, et al. FOCUS 1: a randomized, double-blinded, multicentre, Phase III trial of the efficacy and safety of ceftaroline fosamil versus ceftriaxone in community-acquired pneumonia. J Antimicrob Chemother. 2011;66 (Suppl 3:iii):19-32.

4. Low DE, File TM Jr, Eckburg PB, et al. FOCUS 2: a randomized, double-blinded, multicentre, Phase III trial of the efficacy and safety of ceftaroline fosamil versus ceftriaxone in community-acquired pneumonia. J Antimicrob Chemother. 2011;66 (Suppl 3:iii):33-44.

5. Huang X, Jandourek A, Cole P, Friedland D. Current use of ceftaroline for community-acquired bacterial pneumonia (cabp) in us hospitals: length of stay and total cost from the capture study. Chest J. 2013;144:259A-A.

6. Jandourek A, Udeani G, Smith A, Friedland HD. CAPTURE Study experience in patients with community acquired pneumonia due to methicillin-resistant Staphylococcus aureus (MRSA) and treatment with ceftaroline. European Congress on Clinical Microbiology and Infectious Diseases. Berlin, Germany, 2013.

7. Maggiore C, Pasquale T, Cole P, Smith A, Friedland HD. Ceftaroline fosamil for treatment of acute bacterial skin and skin structure infections or community-acquired bacterial pneumonia in patients with renal insufficiency: CAPTURE study experience. American Society of Health-System Pharmacists' Midyear Meeting. Orlando: American Society of Health-System Pharmacists; 2013.

8. Maggiore C, Pasquale T, Jandourek A, Smith A, Friedland HD. Experience with ceftaroline fosamil 
as monotherapy and combination therapy with vancomycin in acute bacterial skin and skin structure infections and community-acquired bacterial pneumonia. ASHP Midyear Meeting 2013 Orlando, FL American Society of Health-System Pharmacists; 2013. p. 5-112.

9. Udeani G, Evans J, Jandourek A, Friedland HD. Ceftaroline fosamil for the treatment of community-acquired bacterial pneumonia (CABP): CAPTURE Year 1 (H 46). American Thoracic Society International Conference. Philadelphia, PA, 2013.

10. Udeani G, Evans J, Jandourek A, Friedland HD. CAPTURE: Ceftaroline fosamil for the treatment of community acquired bacterial pneumonia (CABP): Year 1. A49 community acquired pneumonia and healthcare-associated pneumonia: treatment and outcomes. American Thoracic Society; 2013. p. A1688-A.

11. van Hal SJ, Fowler VG, Jr. Is it time to replace vancomycin in the treatment of methicillinresistant Staphylococcus aureus infections? Clin Infect Dis Off Publ Infect Dis Soc Am. 2013;56: 1779-88.

12. Wunderink RG, Niederman MS, Kollef MH, et al. Linezolid in methicillin-resistant Staphylococcus aureus nosocomial pneumonia: a randomized, controlled study. Clin Infect Dis Off Publ Infect Dis Soc Am. 2012;54:621-9.

13. Mandell LA, Bartlett JG, Dowell SF, et al. Update of practice guidelines for the management of community-acquired pneumonia in immunocompetent adults. Clin Infect Dis Off Publ Infect Dis Soc Am. 2003;37:1405-33.

14. Mandell LA, Wunderink RG, Anzueto A, et al. Infectious Diseases Society of America/American Thoracic Society consensus guidelines on the management of community-acquired pneumonia in adults. Clin Infect Dis Off Publ Infect Dis Soc Am. 2007;44(Suppl 2):S27-72.

15. Antimicrobial hospital-acquired bacterial pneumonia and ventilator-associated bacterial pneumonia: developing drugs for treatment. 2010. http://www. fda.gov/downloads/Drugs/GuidanceComplianceReg ulatoryInformation/Guidances/UCM234907.pdf. Accessed Aug 25, 2011.

16. Guidance for industry. Community-acquired bacterial pneumonia: developing drugs for treatment, draft guidance. Food and Drug
Administration, Center for Drug Evaluation and Research, Washington, DC. 2009. http://www.fda. gov.elibrary.amc.edu/downloads/Drugs/Guidance ComplianceRegulatoryInformation/Guidances/ucm 123686.pdf. Accessed Aug 8, 2014.

17. Pertel PE, Bernardo P, Fogarty C, et al. Effects of prior effective therapy on the efficacy of daptomycin and ceftriaxone for the treatment of community-acquired pneumonia. Clin infect Dis Off Publ Infect Dis Soc Am. 2008;46:1142-51.

18. Bartlett JG. Diagnostic tests for agents of community-acquired pneumonia. Clin infect Dis Off Publ Infect Dis Soc Am. 2011;52(Suppl 4): S296-304.

19. Fine MJ, Auble TE, Yealy DM, et al. A prediction rule to identify low-risk patients with communityacquired pneumonia. N Engl J Med. 1997;336: 243-50.

20. National Nosocomial Infections Surveillance (NNIS) System report, data summary from January 1992 through June 2004, issued October 2004. Am J Infect Control 2004;32:470-85.

21. Eckberg PB, Friedland DH, Llorens L, et al. Day 4 clinical response of ceftaroline fosamil versus cefriaxone for community-acquired bacterial pneumonia. Infect Dis Clin Pract. 2012;20:254-60.

22. Moisan H, Pruneau M, Malouin F. Binding of ceftaroline to penicillin-binding proteins of Staphylococcus aureus and Streptococcus pneumoniae. J Antimicrob Chemother. 2010;65:713-6.

23. Zhanel GG, Yachison C, Nichol K, et al. Assessment of the activity of ceftaroline against clinical isolates of penicillin-intermediate and penicillin-resistant Streptococcus pneumoniae with elevated MICs of ceftaroline using an in vitro pharmacodynamic model. J Antimicrob Chemother. 2012;67:1706-11.

24. Ramani A, Udeani G, Evans J, et al. Contemporary use of ceftaroline fosamil for the treatment of community-acquired bacterial pneumonia: CAPTURE study experience. J Chemother. 2014 (1973947814Y0000000184).

25. van Hal SJ, Paterson DL. Systematic review and meta-analysis of the significance of heterogeneous vancomycin-intermediate Staphylococcus aureus isolates. Antimicrob Agents Chemother. 2011;55: 405-10. 